PROCEEDINGS OF THE

AMERICAN MATHEMATICAL SOCIETY

Volume 139, Number 10, October 2011, Pages 3583-3599

S 0002-9939(2011)10782-2

Article electronically published on March 3, 2011

\title{
EIGENFUNCTION ESTIMATES FOR NEUMANN LAPLACIAN AND APPLICATIONS TO MULTIPLIER PROBLEMS
}

\author{
XIANGJIN XU
}

(Communicated by Hart F. Smith)

\begin{abstract}
On compact Riemannian manifolds with boundary, the $L^{\infty}$ estimates and gradient estimates for the eigenfunctions of the Neumann Laplacian are proved. Applying the $L^{p}$ estimates and gradient estimates to multiplier problems on eigenfunction expansions for the Neumann Laplacian, some new estimates for Bochner Riesz means and the sharp Hörmander Multiplier Theorem are obtained.
\end{abstract}

\section{INTRODUCTION}

Let $(M, g)$ be a compact Riemannian manifold of dimension $n \geq 2$ with smooth boundary. We shall consider the Neumann eigenvalue problem:

$$
\left\{\begin{aligned}
\left(\Delta+\lambda^{2}\right) u(x)=0, & x \in M \\
N_{x} \nabla u(x)=0, & x \in \partial M
\end{aligned}\right.
$$

with $\Delta=\Delta_{g}$ being the Laplace-Beltrami operator associated to the metric $g$, and $N_{x}$ being the unit normal field with respect to the metric $g$. Let $0=\lambda_{0}^{2}<\lambda_{1}^{2} \leq$ $\lambda_{2}^{2} \leq \lambda_{3}^{2} \leq \cdots$ denote the spectrum of $-\Delta$, which is discrete and tends to infinity. Let $\left\{e_{j}(x)\right\} \subset L^{2}(M)$ be an associated real orthonormal eigenfunction, and let

$$
e_{j}(f)(x)=e_{j}(x) \int_{M} f(y) e_{j}(y) d y
$$

be the projection onto the $j$-th eigenspace. Here and in what follows $d y$ denotes the volume element associated with the metric $g$. Define the unit band spectral clusters $\chi_{\lambda}$ as

$$
\chi_{\lambda} f=\sum_{\lambda_{j} \in[\lambda, \lambda+1)} e_{j}(f) .
$$

The first part of this paper is to study the $L^{\infty}$ estimates and gradient estimates for spectral clusters $\chi_{\lambda}$ of the Neumann Laplacian. The study of $L^{p}$ estimates for

Received by the editors May 11, 2010 and, in revised form, August 26, 1010.

2010 Mathematics Subject Classification. Primary 35P20, 35J25, 58J05, 58J32, 58J40, 35P15, 35J05.

Key words and phrases. $L^{\infty}$ estimate, gradient estimate, spectral cluster, Neumann Laplacian, Bochner Riesz means, Hörmander Multiplier Theorem.

The author's research was supported by the National Science Foundation under grants DMS0602151 and DMS-0852507.

(C)2011 American Mathematical Society Reverts to public domain 28 years from publication 
the eigenfunctions on compact manifolds has a long history. In the case of manifolds without boundary, the most general results of the form

$$
\left\|\chi_{\lambda} f\right\|_{p} \leq C \lambda^{\sigma(p)}\|f\|_{2}, \sigma(p)=\max \left\{\frac{n-1}{2}-\frac{n}{p}, \frac{n-1}{2}\left(\frac{1}{2}-\frac{1}{p}\right)\right\}, p \geq 2,
$$

were proved by Sogge in [17, which are sharp for all compact manifolds (see [17, [18]). The special case of (2) when $p=\infty$ can be proved using the estimates of Hörmander in [7] that proved the sharp Weyl formula for general self-adjoint elliptic operators on manifolds without boundary. Recently, in the case of manifolds without boundary, Sogge and Zelditch in [20] proved that for generic metrics on any compact manifold without boundary one has the bounds $\left\|e_{j}\right\|_{\infty}=o\left(\lambda_{j}^{(n-1) / 2}\right)$ for $L^{2}$-normalized eigenfunctions. In the case of manifolds with boundary, the issue of spectral cluster estimates is considerably more intricate. For example, the Rayleigh whispering gallery modes provide examples of spectral clusters which concentrate in a $\lambda^{-\frac{2}{3}} \times \lambda^{-\frac{n-2}{2}}$ neighborhood of a boundary geodesic (see [5]). These examples show that one cannot achieve linear spectral cluster estimates better than

$$
\left\|\chi_{\lambda}\right\|_{L^{2} \rightarrow L^{p}} \lesssim\left\{\begin{aligned}
\lambda^{\left(\frac{2}{3}+\frac{n-2}{2}\right)\left(\frac{1}{2}-\frac{1}{p}\right)}, & 2 \leq p \leq \frac{6 n+4}{3 n-4}, \\
\lambda^{n\left(\frac{1}{2}-\frac{1}{p}\right)-\frac{1}{2}}, & \frac{6 n+4}{3 n-4} \leq p \leq \infty .
\end{aligned}\right.
$$

It should be noted that the Rayleigh mode construction can only be done near a portion of the boundary that is strictly convex. When the boundary is strictly concave, the bounds (2) actually hold, as was shown by Grieser in [5] for $n=2$, and subsequently for all $n$ by Smith and Sogge in [14. Recently, without assuming the concave boundary, for the special case of (3) with $p=\infty$ using the estimates of the sharp Weyl formula and a maximum principle argument, Grieser in [6] proved the $L^{\infty}$ estimates for a single eigenfunction of the Dirichlet and Neumann Laplacian,

$$
\left\|e_{j}(f)\right\|_{\infty} \lesssim \lambda_{j}^{(n-1) / 2}\|f\|_{2},
$$

and Sogge in [19] proved the $L^{\infty}$ estimate for spectral clusters of the Dirichlet Laplacian,

$$
\left\|\chi_{\lambda} f\right\|_{\infty} \lesssim \lambda^{(n-1) / 2}\|f\|_{2}, \quad \lambda \geq 1
$$

which are sharp for instance when $M$ is the upper hemisphere of $S^{n}$ with standard metric. The estimates (3) were recently proven for dimension $n=2$ by Smith and Sogge in 15, along with partial results in higher dimensions. The question of whether or not the estimates (3) hold in general in higher dimensions remains open. More literature discussions on $L^{p}$ estimates for spectral clusters could be found in 15 and 18 . Here, following some ideas in $\left[6\right.$ and 19 , we have the $L^{\infty}$ estimates for the spectral clusters $\chi_{\lambda}$ of the Neumann Laplacian as:

Theorem 1.1. Fix a compact Riemannian manifold $(M, g)$ with boundary. Then the $L^{\infty}$ estimates for the spectral clusters $\chi_{\lambda}$ of the Neumann Laplacian are

$$
\left\|\chi_{\lambda} f\right\|_{\infty} \leq C \lambda^{(n-1) / 2}\|f\|_{2}
$$

where $C$ is a uniform constant for all $\lambda \geq 0$.

One important application of $L^{p}$ estimates of spectral clusters is to study the boundedness of Bochner-Riesz means (see 11, [16], 18, 19, etc.). Using a fundamental proposition in [19], we obtain some new estimates for Bochner-Riesz means for eigenfunction expansions from the favorable $L^{p}$ estimates of spectral clusters. 
The next result is to study the gradient estimates for the spectral clusters $\chi_{\lambda}$ of the Neumann Laplacian. In [25], the author proved the gradient estimates of the spectral clusters of the Dirichlet Laplacian, based on the $L^{\infty}$ estimates from [19]. Here, based on the $L^{\infty}$ estimates in Theorem 1.1, we have the gradient estimates for the spectral clusters $\chi_{\lambda}$ of the Neumann Laplacian:

Theorem 1.2. Fix a compact Riemannian manifold $(M, g)$ with boundary. Then the gradient estimates for the spectral clusters $\chi_{\lambda}$ of the Neumann Laplacian are

$$
\left\|\nabla \chi_{\lambda} f\right\|_{\infty} \leq C \lambda^{(n+1) / 2}\|f\|_{2},
$$

where $C$ is a uniform constant for all $\lambda \geq 0$.

Remark 1.1. The motivation to study the gradient estimates, which is one of the essential estimates in the proof of the sharp Hörmander Multiplier Theorem, is that one cannot get the gradient estimates on the eigenfunctions of the Dirichlet Laplacian or the Neumann Laplacian from the standard calculus of pseudodifferential operators as done for the Laplacian on the manifolds without boundary, since $P=\sqrt{-\Delta_{g}}$ for the Dirichlet Laplacian or the Neumann Laplacian is not a pseudo-differential operator any more and one cannot get good estimates on $L^{\infty}$ bounds on $\chi_{\lambda}$ and $\nabla \chi_{\lambda}$ near the boundary only by studying the Hadamard parametrix of the wave kernel as for compact manifolds without boundary in [11] and [18].

Applying the new $L^{\infty}$ estimates and gradient estimates for spectral clusters $\chi_{\lambda}$, we shall prove the sharp Hörmander Multiplier Theorem for eigenfunction expansions of the Neumann Laplacian. Given a bounded function $m(\lambda) \in L^{\infty}(\mathbf{R})$, one can define a Hörmander multiplier operator $m(P)$ with $P=\sqrt{-\Delta}$ by

$$
m(P) f=\sum_{j=1}^{\infty} m\left(\lambda_{j}\right) e_{j}(f) ;
$$

such an operator is always bounded on $L^{2}(M)$. However, if one considers any other space $L^{p}(M)$, it is known that some smoothness assumptions on the function $m(\lambda)$ are needed to ensure the boundedness of

$$
m(P): L^{p}(M) \rightarrow L^{p}(M) .
$$

Based on the sharp results on Riesz means ([1], [16], 21]), one introduces the following sharp smoothness assumption on the multiplier function $m(\lambda)$ when one studies the Hörmander multiplier problem:

The sharp smoothness assumption on $m(\lambda)$. Let $L_{s}^{2}(\mathbf{R})$ denote the usual Sobolev space, and fix $\beta \in C_{0}^{\infty}((1 / 2,2))$ satisfying $\sum_{-\infty}^{\infty} \beta\left(2^{j} t\right)=1, \forall t>0$. The multiplier function $m \in L^{\infty}(\mathbf{R})$ satisfies

$$
\sup _{\lambda>0} \lambda^{-1+s}\|\beta(\cdot / \lambda) m(\cdot)\|_{L_{s}^{2}}^{2}=\sup _{\lambda>0}\|\beta(\cdot) m(\lambda \cdot)\|_{L_{s}^{2}}^{2}<\infty, \quad \forall s>n / 2 .
$$

Hörmander [8] first proved the boundedness of the multiplier operator $m(P)$ on $\mathbf{R}^{n}$ under the assumption (8). Stein and Weiss [21] studied the Hörmander Multiplier Theorem for multiple Fourier series, which can be regarded as the case on the flat torus $\mathrm{T}^{n}$. Seeger and Sogge 11 and Sogge [18 proved the boundedness of $m(P)$ on $L^{p}(M)$ for compact manifolds without boundary under the assumption (8), where they used the parametrix of the wave kernel of $m(P)$ to get the required 
estimates on the integral kernel of $m(P)$. In 2 Duong-Ouhabaz-Sikora gave another proof of the Hörmander Multiplier Theorem using heat kernel methods and the $L^{\infty}$ bounds of $\chi_{\lambda}$ on manifolds without boundary. In [24] the author gave a new proof of the boundedness of $m(P)$ on $L^{p}(M)$ using $L^{\infty}$ estimates on $\chi_{\lambda} f$ and $\nabla \chi_{\lambda} f$ but without using the parametrix of the wave kernel of $m(P)$. As one knows, the parametrix construction of the wave kernel does not work well for general compact manifolds with boundary unless one further assumes that the boundary is geodesically concave, which means that the method in [11 could not apply to a manifold with boundary but the method in [24] works. In [25] the author proved the sharp Hörmander Multiplier Theorem using $L^{\infty}$ estimates on $\chi_{\lambda} f$ and $\nabla \chi_{\lambda} f$ for the Dirichlet Laplacian. Using the $L^{\infty}$ estimates (4) and gradient estimates (5) for the Neumann Laplacian and the ideas from [24, 25], we prove the sharp Hörmander Multiplier Theorem for eigenfunction expansions for the Neumann Laplacian:

Theorem 1.3. Let $m \in L^{\infty}(\mathbf{R})$ satisfy the sharp smoothness assumption (8) on the multiplier function $m(\lambda)$. Then, for $1<p<\infty$, one has the boundedness of the Hörmander multiplier operator $m(P)$ on $L^{p}(M)$ :

$$
\|m(P) f\|_{L^{p}(M)} \lesssim\|f\|_{L^{p}(M)}, \quad 1<p<\infty .
$$

For $p=1$, there is the weak-type $(1,1)$ estimate:

$$
\mu\{x:|m(P) f(x)|>\alpha\} \lesssim \alpha^{-1}\|f\|_{L^{1}}, \quad \forall \alpha>0 .
$$

The outline of this paper is the following: Section 2 will give the proof of the $L^{\infty}$ estimates for the spectral clusters $\chi_{\lambda}$ of the Neumann Laplacian and some applications for the new estimates for Bochner-Riesz means. Section 3 will give the proof of the gradient estimates for the spectral clusters $\chi_{\lambda}$ and prove the sharp Hörmander Multiplier Theorem for the Neumann Laplacian.

In what follows we shall use the convention that $C$ will denote a constant that is not necessarily the same at each occurrence.

\section{2. $L^{\infty}$ Estimates And BochneR-Riesz Means}

Motivated by [19, the purpose of this section is to give a simple proof of sharp $L^{\infty}$ estimates for the spectral clusters $\chi_{\lambda}$ of the Neumann Laplacian on smooth compact Riemannian manifolds $(M, g)$ of dimension $n \geq 2$ with boundary $\partial M$ and then to use these estimates to prove new estimates for Bochner-Riesz means in this setting as Sogge [19] did for the Dirichlet Laplacian.

Here and in the next section we use the geodesic normal coordinates with respect to the boundary. We can find a small constant $c>0$ so that the map $\left(x^{\prime}, x_{n}\right) \in$ $\partial M \times[0, c) \rightarrow M$, sending $\left(x^{\prime}, x_{n}\right)$ to the endpoint $x$, of the geodesic of length $x_{n}$ which starts at $x^{\prime} \in \partial M$ and is perpendicular to $\partial M$ is a local diffeomorphism. Under the geodesic coordinates $x=\left(x_{1}, \ldots, x_{n-1}, x_{n}\right)$, the Riemannian metric has the form

$$
\sum_{i, j=1}^{n} g_{i j}(x) d x^{i} d x^{j}=\left(d x_{n}\right)^{2}+\sum_{i, j=1}^{n} g_{i j}(x) d x^{i} d x^{j},
$$

and the Laplacian can be written as

$$
\Delta_{g}=\sum_{i, j=1}^{n} g^{i j}(x) \frac{\partial^{2}}{\partial x_{i} \partial x_{j}}+\sum_{i=1}^{n} b_{i}(x) \frac{\partial}{\partial x_{i}},
$$


where $\left(g^{i j}(x)\right)_{1 \leq i, j \leq n}$ is the inverse matrix of $\left(g_{i j}(x)\right)_{1 \leq i, j \leq n}$, and $g^{n n}=1, g^{n k}=$ $g^{k n}=0$ for $k \neq n$. Also the $b_{i}(x)$ are $C^{\infty}$ and real-valued. For simplicity of notation, we let $d(x)=\operatorname{dist}(x, \partial M)$, and $d(x)=x_{n}$ near the boundary $\partial M$ under the geodesic normal coordinates.

2.1. $L^{\infty}$ estimates. First we shall prove Theorem 1.1, the $L^{\infty}$ estimates (4) for spectral clusters of the Neumann Laplacian. Note that

$$
\chi_{\lambda} f(x)=\int_{M} \sum_{\lambda_{j} \in[\lambda, \lambda+1)} e_{j}(x) e_{j}(y) f(y) d y
$$

therefore, by the converse to Schwarz's inequality and orthogonality, one has the bounds (4) at a given point $x$ for all $f$ if and only if

$$
\sum_{\lambda_{j} \in[\lambda, \lambda+1)} e_{j}(x)^{2} \lesssim \lambda^{n-1}
$$

The proof of Theorem 1.1 is reduced to the following two propositions:

Proposition 2.1. Fix the compact manifold $(M, g)$ with boundary, for a given small constant $\epsilon>0$, there is a uniform constant $C_{\epsilon}$ so that for $\lambda \geq 0$,

$$
\sum_{\lambda_{j} \in[\lambda, \lambda+1)} e_{j}(x)^{2} \leq C_{\epsilon} \lambda^{n-1},
$$

for any interior point $x$ satisfying $d(x) \geq \epsilon \lambda^{-1}$.

Proposition 2.2. Fix $(M, g)$ as above. Then for large $\lambda$ we have

$$
\max _{\left\{x: d(x) \leq \frac{1}{2}(\lambda+1)^{-1}\right\}} \sum_{\lambda_{j} \in[\lambda, \lambda+1)} e_{j}(x)^{2} \leq 4 \max _{\left\{x: d(x)=\frac{1}{2}(\lambda+1)^{-1}\right\}} \sum_{\lambda_{j} \in[\lambda, \lambda+1)}\left(e_{j}(x)\right)^{2} .
$$

Proof of Proposition 2.1. As in the proof of Proposition 2.1 in [19] for the interior $L^{\infty}$ estimates for spectral clusters of the Dirichlet Laplacian, we shall see that the estimate for the Neumann Laplacian in this proposition is an immediate consequence of a result similar to Theorem 17.5.10 in [8], which in turn is based on earlier work of Seeley in 12 .

Assume that the local coordinates have been chosen to the geodesic normal coordinates with respect to the boundary the same as in Theorem 17.5.10 in Hörmander [8], and define the spectral function

$$
\begin{aligned}
e(x, y, \lambda) & =\sum_{\lambda_{j} \leq \lambda} e_{j}(x) e_{j}(y) \\
e(x, \lambda) & =(2 \pi)^{-n} \int_{\left\{\xi \in \mathbf{R}^{n}|| \xi \mid \leq \lambda\right\}}\left(1-e^{i 2 d(x) \xi_{n}}\right) d \xi .
\end{aligned}
$$

Then one has the following result for the Neumann Laplacian the same as in Theorem 17.5.10 in Hörmander [8]:

For the spectral function $e(x, y, \lambda)$ of the Neumann problem on $M$, we have

$$
\left|\sum_{\lambda_{j} \leq \lambda}\left(e_{j}(x)\right)^{2}-e(x, \lambda)\right| \leq C \lambda\left(\lambda+d(x)^{-1}\right)^{n-2}, \forall \lambda \geq 0 .
$$


Since $\lambda\left(\lambda+d(x)^{-1}\right)^{n-2}=O\left(\lambda^{n-1}\right), \forall x$ satisfying $d(x) \geq \epsilon \lambda^{-1}$, and

$$
|e(x, \lambda+1)-e(x, \lambda)| \leq C_{\epsilon} \lambda^{n-1}, \quad \forall x \text { with } d(x) \geq \epsilon \lambda^{-1},
$$

the estimate (11) implies Proposition 2.1.

One can see that the proof of estimate (11) for the Neumann Laplacian is a straightforward modification of the proof of Theorem 17.5.10 in Hörmander [8] for the Dirichlet Laplacian. We just give an outline here. When $d(x)>c>0$ for some fixed small constant $c$, this is a consequence of Theorem 7.2 in [12], where Seeley proved:

There are constants $C$ and $\alpha>0$ such that for $\lambda>1$,

$$
\left|\sum_{\lambda_{j} \leq \lambda}\left(e_{j}(x)\right)^{2}-e(x, \lambda)\right| \leq C\left[d(x)^{\alpha-1} \lambda^{n-1}+d(x)^{-3 / 2} \lambda^{n-3 / 2}\right] .
$$

This is true for both the Dirichlet Laplacian and the Neumann Laplacian.

When $d(x) \leq c$, we will use the Tauberian Lemma (Lemma 17.5.6 in Hörmander [8]) with $a=1 / c$ and define

$$
\begin{aligned}
\mu(\lambda) & =\frac{1}{2} \operatorname{sign}(\lambda) \sum_{\lambda_{j} \leq|\lambda|}\left(e_{j}(x)\right)^{2}, \\
\nu(\lambda) & =\frac{1}{2} \operatorname{sign}(\lambda) e(x,|\lambda|)
\end{aligned}
$$

for $\lambda \in \mathbf{R}$. By the definition of $e(x, \lambda)$, we have

$$
|d e(x, \lambda) / d \lambda| \leq C \lambda^{n-1} .
$$

This proves the first part of assumption (17.5.13) of the Tauberian Lemma 17.5.6 in [8]. The same as in the proof of Theorem 15.5.10 in [8] for the Dirichlet Laplacian, the estimate (11) will follow if we prove that

$$
\left|(d \mu-d \nu) * \phi_{a}(\lambda)\right| \leq C\left(|\lambda|+d(x)^{-1}\right)^{n-2},
$$

where $\phi_{a}(\lambda)=\frac{1}{a} \phi(\lambda / a)$ for $a>0$, and $\phi$ is a positive function in $C^{\infty}(\mathbf{R})$ with $\int_{\mathbf{R}} \phi(\lambda) d \lambda=1$ and the Fourier transform $\hat{\phi}$ with support in $(-1,1)$. We can show that the above estimate for the Neumann Laplacian is the same as for Theorem 17.5.10 in [8]. The only difference is that we should replace those estimates for the Dirichlet wave kernel used in the proof of Theorem 17.5.10 in [8] by these corresponding estimates for the Neumann wave kernel with some different constants, which can be found in Seeley's paper [12.

Proof of Proposition 2.2. Our proof follows the ideas of the proof of Proposition 2.4 in [19. In what follows we shall assume that $\lambda$ is large enough and assume further that $\operatorname{spec}\left(\sqrt{-\Delta_{g}}\right) \cap[\lambda, \lambda+1] \neq \emptyset$, and consider the function

$$
H(x)=\frac{1}{w(x)^{2}} \sum_{\lambda_{j} \in[\lambda, \lambda+1)} e_{j}(x)^{2},
$$

where $w(x)=1-(\lambda+1)^{2} x_{n}^{2}$. We shall show that the maximum value of $H(x)$ must be at the boundary of the $\lambda^{-1}$ strip, and furthermore, we show that the outward normal derivatives of $H(x)$ on the boundary $\partial M$ must be strictly positive. 
Suppose that $H(x)$ has a maximum at an interior point $x=x_{0}$ in the strip $\left\{x \in M: 0 \leq x_{n} \leq \frac{1}{2}(\lambda+1)^{-1}\right\}$. Then by the Cauchy-Schwarz inequality,

$$
v(x)=\sum_{\lambda_{j} \in[\lambda, \lambda+1)} \frac{e_{j}\left(x_{0}\right)}{w\left(x_{0}\right)} \frac{e_{j}(x)}{w(x)}
$$

must have a positive maximum at $x=x_{0}$. Note that in the strip $\{x \in M: 0 \leq$ $\left.x_{n} \leq \frac{1}{2}(\lambda+1)^{-1}\right\}$ we have

$$
\begin{aligned}
\left(\Delta+\lambda_{j}^{2}\right) w & =-2(\lambda+1)^{2}-2 b_{n}(x) x_{n}(\lambda+1)^{2}+\lambda_{j}\left(1-(\lambda+1)^{2} x_{n}^{2}\right) \\
& \leq-\frac{(\lambda+1)^{2}}{2}, \quad \text { for } \lambda \leq \lambda_{j} \leq \lambda+1,
\end{aligned}
$$

assuming that $\lambda$ is large enough so that $\left|2 b_{n}(x) x_{n}\right| \leq 1 / 2$ in the strip. Also, in this strip we have that $\frac{1}{2} \leq w(x) \leq 1$.

Define $v_{j}(x)=\frac{e_{j}\left(x_{0}\right)}{w\left(x_{0}\right)} \frac{e_{j}(x)}{w(x)}$ and $v(x)=\sum_{\lambda_{j} \in[\lambda, \lambda+1)} v_{j}(x)$. Note that

$$
\left(\Delta+\lambda_{j}^{2}\right) u_{j}(x)=0,\left.\quad \partial_{n} u_{j}\right|_{\partial M}=0, \quad \text { where } \quad u_{j}(x)=\frac{e_{j}\left(x_{0}\right)}{w\left(x_{0}\right)} e_{j}(x) .
$$

A direct computation shows that for a given $j$ we have

$$
\begin{aligned}
0 & =\frac{1}{w(x)}\left(\Delta+\lambda_{j}^{2}\right) u_{j}(x) \\
& =\sum_{k, l=1}^{n} g^{k l}(x) \partial_{k} \partial_{l} v_{j}+\sum_{k=1}^{n}\left(\frac{2}{w} \sum_{l=1}^{n} g^{k l} \partial_{l} w+b_{k}\right) \partial_{k} v_{j}+\frac{v_{j}}{w}\left(\Delta+\lambda_{j}^{2}\right) w .
\end{aligned}
$$

Therefore, summing over $\lambda_{j} \in[\lambda, \lambda+1)$, we get

$$
\sum_{k, l=1}^{n} g^{k l}(x) \partial_{k} \partial_{l} v+\sum_{k=1}^{n}\left(\frac{2}{w} \sum_{l=1}^{n} g^{k l} \partial_{l} w+b_{k}\right) \partial_{k} v=-\sum_{\lambda_{j} \in[\lambda, \lambda+1)} \frac{v_{j}}{w}\left(\Delta+\lambda_{j}^{2}\right) w .
$$

In particular, at point $x=x_{0}$, we have

$$
\begin{aligned}
& \sum_{k, l=1}^{n} g^{k l}\left(x_{0}\right) \partial_{k} \partial_{l} v\left(x_{0}\right)+\sum_{k=1}^{n}\left(\frac{2}{w} \sum_{l=1}^{n} g^{k l}\left(x_{0}\right) \partial_{l} w\left(x_{0}\right)+b_{k}\left(x_{0}\right)\right) \partial_{k} v\left(x_{0}\right) \\
& \quad=-\frac{1}{w\left(x_{0}\right)} \sum_{\lambda_{j} \in[\lambda, \lambda+1)}\left(\frac{e_{j}\left(x_{0}\right)}{w\left(x_{0}\right)}\right)^{2}\left(\Delta+\lambda_{j}^{2}\right) w\left(x_{0}\right) \\
& \quad \geq \frac{(\lambda+1)^{2}}{2 w\left(x_{0}\right)} \sum_{\lambda_{j} \in[\lambda, \lambda+1)}\left(\frac{e_{j}\left(x_{0}\right)}{w\left(x_{0}\right)}\right)^{2}>0 .
\end{aligned}
$$

But this is impossible since $v(x)$ have a positive maximum at $x_{0}$, which implies that $\partial_{k} v\left(x_{0}\right)=0$ for every $k$, and $\sum_{k, l=1}^{n} g^{k l}\left(x_{0}\right) \partial_{k} \partial_{l} v\left(x_{0}\right) \leq 0$. Thus, we conclude that the function $H(x)$ cannot have a maximum at an interior point of the strip $\left\{x \in M: 0 \leq x_{n} \leq \frac{1}{2}(\lambda+1)^{-1}\right\}$.

Next we shall prove that the function $H(x)$ cannot have a maximum value of the strip on $\partial M$. Suppose that there is a maximum at the boundary point $x_{0}=\left(x^{\prime}, 0\right)$ on $\partial M$. Then by the same argument as above, we have that $v(x)$ has a positive 
maximum at $x_{0}$ on $\partial M$, in our local coordinates, which means that $\partial_{k} v\left(x_{0}\right)=0$ for every $k \leq n-1$, and $\sum_{k, l=1}^{n-1} g^{k l}\left(x_{0}\right) \partial_{k} \partial_{l} v\left(x_{0}\right) \leq 0$. We also have

$$
\begin{aligned}
\partial_{n} v(x) & =\left(\partial_{n} \frac{1}{w(x)}\right) \sum_{\lambda_{j} \in[\lambda, \lambda+1)} u_{j}(x)+\frac{1}{w(x)} \sum_{\lambda_{j} \in[\lambda, \lambda+1)} \partial_{n} u_{j}(x) \\
& =-\frac{2(\lambda+1)^{2} x_{n}}{w(x)^{2}} \sum_{\lambda_{j} \in[\lambda, \lambda+1)} u_{j}(x)+\frac{1}{w(x)} \sum_{\lambda_{j} \in[\lambda, \lambda+1)} \partial_{n} u_{j}(x) .
\end{aligned}
$$

Given the Neumann boundary condition, we have $\partial_{n} v\left(x_{0}\right)=0$. For our local coordinates, we have $g^{n n}=1$, and $g^{n k}=g^{k n}=0$ for $k \neq n$. Hence from (12), we have

$$
\partial_{n}^{2} v\left(x_{0}\right)=\frac{(\lambda+1)^{2}}{2} \sum_{\lambda_{j} \in[\lambda, \lambda+1)} e_{j}^{2}\left(x_{0}\right)>0 .
$$

But it cannot be true, since in local coordinates we have that $v\left(x_{0}^{\prime}, x_{n}\right)$ gets its maximum at $x_{0}=\left(x_{0}^{\prime}, 0\right)$ from our assumption and $\partial_{n} v\left(x_{0}\right)=0$, which implies $\partial_{n}^{2} v\left(x_{0}\right) \leq 0$. This is a contradiction.

From above and our lower bound for $w$, we get that

$$
\max _{\left\{x: d(x) \leq \frac{1}{2}(\lambda+1)^{-1}\right\}} \sum_{\lambda_{j} \in[\lambda, \lambda+1)}\left(e_{j}(x)\right)^{2} \leq 4 \max _{\left\{x: d(x)=\frac{1}{2}(\lambda+1)^{-1}\right\}} \sum_{\lambda_{j} \in[\lambda, \lambda+1)}\left(e_{j}(x)\right)^{2},
$$

as desired, which completes the proof of Proposition 2.2.

Remark 2.1. One may see that these $L^{\infty}$ estimates can also be obtained from the general $L^{p}$ estimates on spectral clusters at $p=\infty$ as in the recent Smith and Sogge paper [15. The author first obtained the estimates of Theorem 1.1 in his Ph.D. thesis 23 before the paper 15] came out. Unlike the $L^{\infty}$ estimates for the Dirichlet Laplacian in [19, where the interior $L^{\infty}$ estimates directly come from Theorem 17.5.10 in [8, which is for the Dirichlet Laplacian only, here we need to prove the estimate (11) for the Neumann Laplacian, which is not available in the literature yet. We prove the estimate (11) using some estimates for the spectral function from Seeley in [12] for the Neumann Laplacian.

2.2. Bochner-Riesz means. Next we shall see how favorable estimates for the spectral clusters of the Laplacian imply sharp estimates for Bochner-Riesz means for eigenfunction expansions. Recall that the Bochner-Riesz means of index $\delta \geq 0$ for eigenfunction expansions are defined by

$$
S_{\lambda}^{\delta} f=\sum_{\lambda_{j} \leq \lambda}\left(1-\frac{\lambda_{j}^{2}}{\lambda^{2}}\right)^{\delta} e_{j}(f) .
$$

It is known that a necessary condition for these operators to be uniformly bounded on $L^{p}(M)$ for a given $1 \leq p \leq \infty, p \neq 2$, is that

$$
\delta(p)=\max \left\{n\left|\frac{1}{2}-\frac{1}{p}\right|-\frac{1}{2}, 0\right\} .
$$

Note that when $p \geq \frac{2 n+2}{n-1}$, one has $\delta(p)=\sigma(p)$, where $\sigma(p)$ is the exponent in (2). Using this fact, Sogge in [16] used the estimates (2) to prove the first sharp estimates 
for Bochner-Riesz means on compact Riemannian manifolds without boundary. Specifically, it was shown that one has the uniform bounds

$$
\left\|S_{\lambda}^{\delta} f\right\|_{p} \lesssim\|f\|_{p}, \quad \forall \delta>\delta(p)
$$

for a given $p \in\left[1, \frac{2 n+2}{n+3}\right] \cup\left[\frac{2 n+2}{n-1}, \infty\right]$. Earlier, weaker results were due to many people, including Hörmander in [7].

In [16] the Tauberian arguments behind the proof of the sharp Weyl formula were adapted to show that one could write $S_{\lambda}^{\delta}=\tilde{S}_{\lambda}^{\delta}+R_{\lambda}^{\delta}$, where the remainder term $R_{\lambda}^{\delta}$ could be controlled by the $L^{p}$ estimates of spectral clusters, while the other piece, $\tilde{S}_{\lambda}^{\delta}$, could be estimated by computing its kernel explicitly via the Hadamard parametrix and then estimating the resulting integral operator using straightforward adaptations of the arguments for the Euclidean case. In the setting of manifolds with boundary, this approach does not seem to work since the known parametrices for the wave equation do not seem strong enough unless one assumes that the boundary is geodesically concave. For the Dirichlet Laplacian, in [19] Sogge got around this fact by simplifying the earlier arguments and showed that estimates for Bochner-Riesz operators just follow from $L^{\infty}$ estimates of spectral clusters and the finite propagation speed of solutions of the Dirichlet wave equation. Specifically, in [19] Sogge proved the following basic proposition:

Proposition 2.3 (Proposition 3.5 in [19]). Assume that for a given $1 \leq p<2$ one has the uniform bounds

$$
\left\|\chi_{\lambda} f\right\|_{2} \leq C \lambda^{\delta(p)}\|f\|_{p}, \quad \lambda \geq 0
$$

where $\delta(p)$ is as in (14). Then for a given $\delta>\delta(p)$ there is a uniform constant $C_{\delta}$ so that

$$
\left\|S_{\lambda}^{\delta} f\right\|_{p} \leq C_{\delta}\|f\|_{p}, \quad \lambda \geq 0
$$

The proof of this proposition in [19] made use of the desired $L^{p}$ estimates (15) of spectral clusters and the finite propagation speed of solutions of the Dirichlet wave equation. Hence Proposition 2.3 will still be true as long as we have the desired $L^{p}$ estimates (15) of spectral clusters and the finite propagation speed property for solutions of the corresponding wave equation in other settings, such as the Neumann Laplacian on compact manifolds with boundary, the Dirichlet Laplacian and the Neumann Laplacian on compact manifolds with strictly geodesic concave boundary [5] [14, and the Laplacian on boundaryless manifolds with $C^{1,1}$ metric [13].

For the manifolds with general boundary, since one only knows that the desired bounds for spectral clusters (2) hold for $p=\infty$, one can only at this stage prove the sharp estimates for Bochner-Riesz means when $p=1$ or $p=\infty$. In 19 Sogge proved such estimates for Bochner-Riesz means of eigenfunctions of the Dirichlet Laplacian. Here we have the following new estimates for Bochner-Riesz means for the Neumann Laplacian from Proposition 2.3:

Theorem 2.1. Fix a smooth compact Riemannian manifold with boundary $(M, g)$ of dimension $n \geq 2$. Then if $\delta>(n-1) / 2$, one has the uniform bounds

$$
\left\|S_{\lambda}^{\delta} f\right\|_{p} \leq C\|f\|_{p}
$$

for every $1 \leq p \leq \infty$. 
By interpolating with the trivial estimate for $p=2$ and using duality, one gets the bounds (17) from the special case where $p=1$, which is sharp. However, the bounds for $1<p<\infty$ are certainly not sharp.

For the Dirichlet Laplacian and the Neumann Laplacian on compact manifolds with strictly geodesic concave boundary, from [5] and [14, we know that the sharp $L^{p}$ estimates (2) are still true, which means we have the desired bounds for spectral clusters (2) holding for $1 \leq p \leq \frac{2 n+2}{n+3}$. Hence we have the following sharp estimates for Bochner-Riesz means in this setting from Proposition 2.3:

Theorem 2.2. Fix a smooth compact Riemannian manifold with strictly geodesic concave boundary. For the Bochner-Riesz means $S_{\lambda}^{\delta}$ of the Dirichlet Laplacian or the Neumann Laplacian, one has the uniform bounds

$$
\left\|S_{\lambda}^{\delta} f\right\|_{p} \lesssim\|f\|_{p}, \quad \forall \delta>\delta(p)
$$

for a given $p \in\left[1, \frac{2 n+2}{n+3}\right] \cup\left[\frac{2 n+2}{n-1}, \infty\right]$.

For the Laplacian on boundaryless manifolds with $C^{1,1}$ metric, from 13 we know that the sharp $L^{p}$ estimates (2) are still true, which means that we have the desired bounds for spectral clusters (2) holding for $1 \leq p \leq \frac{2 n+2}{n+3}$. Hence we have the following sharp estimates for Bochner-Riesz means in this setting from Proposition 2.3:

Theorem 2.3. Fix a boundaryless manifold with $C^{1,1}$ metric. For the BochnerRiesz means $S_{\lambda}^{\delta}$, one has the uniform bounds

$$
\left\|S_{\lambda}^{\delta} f\right\|_{p} \lesssim\|f\|_{p}, \quad \forall \delta>\delta(p)
$$

for a given $p \in\left[1, \frac{2 n+2}{n+3}\right] \cup\left[\frac{2 n+2}{n-1}, \infty\right]$.

\section{Gradient estimates and Hörmander multiplier theorem}

3.1. Gradient estimates. We shall prove Theorem 1.2 by the maximum principle according to two different cases: for the interior points with $\operatorname{dist}(x, \partial M) \geq \epsilon \lambda^{-1}$ and for the points on the strip near the boundary with $\operatorname{dist}(x, \partial M) \leq \epsilon \lambda^{-1}$. Note that

$$
\nabla \chi_{\lambda} f(x)=\int_{M} \sum_{\lambda_{j} \in[\lambda, \lambda+1)} \nabla e_{j}(x) e_{j}(y) f(y) d y ;
$$

therefore, by the converse to Schwarz's inequality and orthogonality, one has the bounds (5) at a given point $x$ if and only if

$$
\sum_{\lambda_{j} \in[\lambda, \lambda+1)}\left|\nabla e_{j}(x)\right|^{2} \leq C^{2} \lambda^{n+1} .
$$

First we have the following gradient estimate for the interior points with $\operatorname{dist}(x, \partial M)$ bounded below by $\lambda^{-1}$ :

Proposition 3.1. For the Riemannian manifold $(M, g)$ with boundary, for a given constant $\epsilon>0$, there is a uniform constant $C_{\epsilon}$ so that for $\lambda \geq 0$ we have the gradient estimate

$$
\left|\nabla \chi_{\lambda} f(x)\right| \leq C_{\epsilon} \lambda^{(n+1) / 2}|| \chi_{\lambda} f \|_{2}, \quad \text { for } \operatorname{dist}(x, \partial M) \geq \epsilon(\lambda+1)^{-1} .
$$


The proof of this proposition is the same as the proof of Proposition 2.1 in 25] using the ideas of interior gradient estimates for Poisson's equation in [4, while we use the $L^{\infty}$ estimates (4) for the Neumann Laplacian to replace the $L^{\infty}$ estimates for the Dirichlet Laplacian in the proof of Proposition 2.1 in [25].

Next we show the gradient estimates on the $\frac{1}{4} \lambda^{-1}$ strip of the boundary.

Proposition 3.2. Fix $(M, g)$ as above. Then for large $\lambda$ we have the gradient estimate

$$
\left|\nabla \chi_{\lambda} f(x)\right| \leq C \lambda^{(n+1) / 2}|| \chi_{\lambda} f \|_{2}, \forall 0 \leq \operatorname{dist}(x, \partial M) \leq \frac{1}{4}(\lambda+1)^{-1} .
$$

Proof. We shall apply the maximum principle to $\sum_{\lambda_{j} \in[\lambda, \lambda+1)}\left|\nabla e_{j}(x)\right|^{2}$ on the $\lambda^{-1}$ boundary strip. First, from the Bochner formulas for the Laplacian, we have

$$
\begin{aligned}
& \frac{1}{2} \Delta_{g} \sum_{\lambda_{j} \in[\lambda, \lambda+1)}\left|\nabla e_{j}(x)\right|^{2} \\
= & \sum_{\lambda_{j} \in[\lambda, \lambda+1)}\left[\left|\operatorname{Hessian}\left(e_{j}\right)\right|^{2}+\left(\nabla e_{j}, \nabla\left(\Delta e_{j}\right)\right)+\operatorname{Ric}\left(\nabla e_{j}, \nabla e_{j}\right)\right] .
\end{aligned}
$$

Since $M$ is a compact manifold, the Ricci curvature is bounded below in all of $M$. Then for large $\lambda$, we have

$$
\begin{aligned}
\frac{1}{2} \Delta_{g} \sum_{\lambda_{j} \in[\lambda, \lambda+1)}\left|\nabla e_{j}(x)\right|^{2} & \geq-\sum_{\lambda_{j} \in[\lambda, \lambda+1)}\left[\lambda_{j}^{2}\left|\nabla e_{j}\right|^{2}-\operatorname{Ric}\left(\nabla e_{j}, \nabla e_{j}\right)\right] \\
& \geq-(\lambda+2)^{2} \sum_{\lambda_{j} \in[\lambda, \lambda+1)}\left|\nabla e_{j}(x)\right|^{2} .
\end{aligned}
$$

Now define a function $w(x)=1-a(\lambda+1)^{2} x_{n}^{2}$ on the $\lambda^{-1}$ strip of the boundary $\left\{x \in M \mid 0 \leq x_{n} \leq \epsilon(\lambda+1)^{-1}\right\}$, and the constants $a$ and $\epsilon$ will be determined below. Set $\frac{1}{2} \leq 1-a \epsilon^{2} \leq w(x) \leq 1$, and

$$
\Delta w(x)=-2 a(\lambda+1)^{2}-2 a b_{n}(x) x_{n}(\lambda+1)^{2} \leq-a(\lambda+1)^{2},
$$

for all points in the strip, here assuming that $\lambda$ is large enough so that $\left|b_{n}(x) x_{n}\right| \leq$ $1 / 2$. Defining $h(x)=\frac{1}{w(x)} \sum_{\lambda_{j} \in[\lambda, \lambda+1)}\left|\nabla e_{j}(x)\right|^{2}$, we have

$$
\begin{aligned}
\Delta_{g} \sum_{\lambda_{j} \in[\lambda, \lambda+1)}\left|\nabla e_{j}(x)\right|^{2} & =w(x) \Delta_{g} h(x)+h(x) \Delta w(x)+2(\nabla h(x), \nabla w(x)) \\
& \geq-2(\lambda+2)^{2} \sum_{\lambda_{j} \in[\lambda, \lambda+1)}\left|\nabla e_{j}(x)\right|^{2} \\
& =-2(\lambda+2)^{2} w(x) h(x) .
\end{aligned}
$$

Dividing both sides by $w(x)$ and applying the estimate on $\Delta_{g} w(x)$, we have

$$
\Delta h(x)+2\left(\nabla h(x), \frac{\nabla w(x)}{w(x)}\right)+(4-a)(\lambda+1)^{2} h(x) \geq 0 .
$$

If we assume $a>4$, by the maximum principle, $h(x)$ achieves its maximum on $\partial\left\{x \in M \mid 0 \leq x_{n} \leq \epsilon(\lambda+1)^{-1}\right\}$. 
Assume that $h(x)$ achieves its nonzero maximum at $x_{0} \in \partial M$. Since we have $\nabla w(x)=0$ for all $x \in \partial M$, then at the point $x_{0} \in \partial M$ where $h(x)$ achieves its maximum, we have

$$
\Delta h\left(x_{0}\right)+(4-a)(\lambda+1)^{2} h\left(x_{0}\right) \geq 0 .
$$

Since $\partial_{k}^{2} h\left(x_{0}\right) \leq 0$ for $k=1,2, \ldots, n-1$, and $h\left(x_{0}\right)>0$, we have $\partial_{n}^{2} h\left(x_{0}\right)>0$. On the other hand, since $w(x)=1, \partial_{n} w(x)=0$ and $\partial_{n} e_{j}(x)=0$ for all $x \in \partial M$, we have $\partial_{k} \partial_{n} e_{j}\left(x_{0}\right)=0$ for $k \leq n-1$,

$$
\begin{aligned}
\partial_{n} h\left(x_{0}\right) & =\partial_{n}\left[\frac{1}{w(x)} \sum_{\lambda_{j} \in[\lambda, \lambda+1)}\left|\nabla e_{j}(x)\right|^{2}\right]_{x=x_{0}} \\
& =\frac{-\partial_{n} w\left(x_{0}\right)}{w\left(x_{0}\right)^{2}} \sum_{\lambda_{j} \in[\lambda, \lambda+1)}\left|\nabla e_{j}\left(x_{0}\right)\right|^{2}+\left.\frac{1}{w\left(x_{0}\right)} \sum_{\lambda_{j} \in[\lambda, \lambda+1)} \partial_{n}\left|\nabla e_{j}(x)\right|^{2}\right|_{x=x_{0}} \\
& =2 \sum_{\lambda_{j} \in[\lambda, \lambda+1)} \sum_{k=1}^{n}\left(\partial_{k} e_{j}\left(x_{0}\right), \partial_{k} \partial_{n} e_{j}\left(x_{0}\right)\right)=0
\end{aligned}
$$

but from $h(x)$ achieving its maximum at $x_{0} \in \partial M$, we have $\partial_{n}^{2} h\left(x_{0}\right) \leq 0$, which is contradicted by the above. Hence we have

$$
\sup _{\left\{x \in M \mid 0 \leq x_{n} \leq \epsilon(\lambda+1)^{-1}\right\}} \sum_{\lambda_{j} \in[\lambda, \lambda+1)}\left|\nabla e_{j}(x)\right|^{2} \leq C \lambda^{n+1} .
$$

Finally we shall determine the constants $a$ and $\epsilon$. From the proof, we need $a>4$ and $a \epsilon^{2} \leq 1 / 2$, which is easy to satisfy; for example, we may let $a=8$ and $\epsilon=1 / 4$.

3.2. Hörmander multiplier theorem. Here we shall prove the sharp Hörmander Multiplier Theorem for the Neumann Laplacian. Since the complex conjugate of $m(\lambda)$ satisfies the same hypotheses (8), we need only to prove Theorem 1.3 for exponents $1<p \leq 2$. This will allow us to exploit orthogonality, and since $m(P)$ is bounded on $L^{2}(M)$, also reduce Theorem 1.3 to show that $m(P)$ is the weak-type $(1,1)$ estimate (10) by the Marcinkiewicz Interpolation Theorem, i.e.,

$$
\mu\{x:|m(P) f(x)|>\alpha\} \lesssim \alpha^{-1}\|f\|_{L^{1}},
$$

where $\mu(E)$ denotes the $d x$ measure of $E \subset M$. Since all the eigenvalues of the Neumann Laplacian are nonnegative, we may assume that $m(t)$ is an even function on $\mathbf{R}$. Then we have

$$
m(P) f(x)=\frac{1}{2 \pi} \int_{\mathbf{R}} \hat{m}(t) e^{i t P} f(x) d t=\frac{1}{\pi} \int_{\mathbf{R}_{+}} \hat{m}(t) \cos (t P) f(x) d t .
$$

Here $P=\sqrt{-\Delta_{g}}$ and the cosine transform $u(t, x)=\cos (t P) f(x)$ is the solution of the following Neumann-Cauchy problem:

$$
\left(\frac{\partial^{2}}{\partial t^{2}}-\Delta_{g}\right) u(t, x)=0, \quad u(0, x)=f(x), u_{t}(0, x)=0, \quad \text { for } x \in M .
$$

We shall use the finite propagation speed of solutions of the wave equation.

The proof of the weak-type $(1,1)$ estimate (10) will involve a splitting of $m(P)$ into two pieces: a main piece which one needs to carefully study, plus a remainder which has the strong $(1,1)$ estimate by using the $L^{\infty}$ estimates for the spectral 
clusters as Sogge did in [18] for manifolds without boundary and as we did in 25] for the Dirichlet Laplacian. Specifically, define $\rho \in C_{0}^{\infty}(\mathbf{R})$ as

$$
\rho(t)=1, \quad \text { for }|t| \leq \frac{\epsilon}{2}, \quad \rho(t)=0, \quad \text { for }|t| \geq \epsilon,
$$

where $\epsilon>0$ is a given small constant related to the manifold, which will be specified later. Write $m(P)=\tilde{m}(P)+r(P)$, where

$$
\begin{aligned}
\tilde{m}(P) & =(m * \check{\rho})(P)=\frac{1}{2 \pi} \int e^{i t P} \rho(t) \hat{m}(t) d t, \\
r(P) & =\left(m *(1-\rho)^{\swarrow}\right)(P)=\frac{1}{2 \pi} \int e^{i t P}(1-\rho(t)) \hat{m}(t) d t .
\end{aligned}
$$

Define for $\lambda=2^{j}, j=1,2, \ldots$,

$$
m_{\lambda}(\tau)=\beta\left(\frac{\tau}{\lambda}\right) m(\tau) .
$$

For the remainder term $r(P) f$, the same as in Part I of the proof of Theorem 1.2 in 24] and [25], we have the strong $(1,1)$ estimate.

\section{Proposition 3.3.}

$$
\|r(P) f\|_{L^{1}} \leq C\|f\|_{L^{1}} .
$$

Next, following the ideas of Part II in the proof of Theorem 1.2 in 24 and [25], we have the weak $(1,1)$ estimate for $\tilde{m}(P)$ :

\section{Proposition 3.4.}

$$
\mu\{x:|\tilde{m}(P) f(x)|>\alpha\} \lesssim \alpha^{-1}|| f \|_{L^{1}}, \quad \forall \alpha>0 .
$$

We give only an outline of the proof here since most of the details of the computations are the same as those in the proof of Theorem 1.2 in 24] and 25]. The weak-type $(1,1)$ estimate on $\tilde{m}(P)$ would follow from the integral operator

$$
\tilde{m}(P) f(x)=\frac{1}{2 \pi} \int_{M}\left\{\int_{\mathbf{R}} \hat{m}(t) \rho(t) \sum_{k \geq 1} e^{i t \lambda_{k}} e_{\lambda_{k}}(x) e_{\lambda_{k}}(y) d t\right\} f(y) d y,
$$

where the kernel $K(x, y)=\sum_{k \geq 1}(m * \check{\rho})\left(\lambda_{k}\right) e_{\lambda_{k}}(x) e_{\lambda_{k}}(y)$ is weak-type $(1,1)$. Now define the dyadic decomposition

$$
K_{\lambda}(x, y)=\int_{\mathbf{R}} \hat{m}_{\lambda}(t) \rho(t) \sum_{k \geq 1} e^{i t \lambda_{k}} e_{\lambda_{k}}(x) e_{\lambda_{k}}(y) d t .
$$

We have $K(x, y)=\sum_{j=1}^{\infty} K_{2^{j}}(x, y)+K_{0}(x, y)$, where $K_{0}$ is bounded and vanishes when $\operatorname{dist}(x, y)$ is larger than a fixed constant. In order to estimate $K_{\lambda}(x, y)$, we make a second dyadic decomposition as follows:

$$
K_{\lambda, l}(x, y)=\int_{\mathbf{R}} \hat{m}_{\lambda}(t) \beta\left(2^{-l} \lambda|t|\right) \rho(t) \sum_{k \geq 1} e^{i t \lambda_{k}} e_{\lambda_{k}}(x) e_{\lambda_{k}}(y) d t,
$$

where we have $K_{\lambda}(x, y)=\sum_{l=-\infty}^{\infty} K_{\lambda, l}(x, y)$. Define

$$
T_{\lambda, l}(P) f(x)=\int_{M} K_{\lambda, l}(x, y) f(y) d y .
$$


From the above two dyadic decompositions, we have

$$
\tilde{m}(P) f(x)=\sum_{k=0}^{\infty} \sum_{l=-\infty}^{\infty} T_{2^{k}, l}(P) f(x) .
$$

Note that because of the support properties of $\rho(t), K_{\lambda, l}(x, y)$ vanishes if $l$ is larger than a fixed multiple of $\log \lambda$. Now we exploit the fact that the finite propagation speed of the wave equation mentioned before implies that the kernels of the operators $T_{\lambda, l}, K_{\lambda, l}$ must satisfy

$$
K_{\lambda, l}(x, y)=0, \quad \text { if } \operatorname{dist}(x, y) \geq C\left(2^{l} \lambda^{-1}\right),
$$

since $\cos (t P)$ will have a kernel that vanishes on this set when $t$ belongs to the support of the integral defining $K_{\lambda, l}(x, y)$. Hence in each of the second sums of (22), there are uniform constants $c, C>0$ such that

$$
c \lambda \operatorname{dist}(x, y) \leq 2^{l} \leq C \lambda
$$

must be satisfied for each $\lambda=2^{k}$, whenever $K_{\lambda, l}$ does not vanish.

Now for each $T_{\lambda, l}(P)$, we have the following two estimates:

Lemma 3.1. $\left\|T_{\lambda, l}(P) f\right\|_{L^{2}(M)} \leq C\left(2^{l}\right)^{-s} \lambda^{n / 2}\|f\|_{L^{1}(M)}$.

Lemma 3.2. $\left\|T_{\lambda, l}(P) g\right\|_{L^{2}(M)} \leq C\left(2^{l}\right)^{-s_{0}} \lambda^{n / 2}\left[\lambda \max _{y, y_{0} \in \Omega} \operatorname{dist}\left(y, y_{0}\right)\right]\|g\|_{L^{1}(\Omega)}$ where $\Omega=\operatorname{support}(g), \int_{\Omega} g(y) d y=0$ and $n / 2<s_{0}<\min \{s, n / 2+1\}$.

The proofs of these lemmas are the same as the estimates $(a),(b)$ on page 470 in [25, where we use the key observation (23) to prove Lemma 3.2. Next we use Lemma 3.1 and Lemma 3.2 to prove Proposition 3.4. We let

$$
f(x)=g(x)+\sum_{k=1}^{\infty} b_{k}(x):=g(x)+b(x)
$$

be the Calderón-Zygmund decomposition of $f \in L^{1}(M)$ at the level $\alpha$ using the same idea as in Lemma 0.2.7 in [18. Letting $Q_{k} \supset \operatorname{supp}\left(b_{k}\right)$ be the cube associated to $b_{k}$ on $M$, we have

$$
\|g\|_{L^{1}}+\sum_{k=1}^{\infty}\left\|b_{k}\right\|_{L^{1}} \leq 3\|f\|_{L^{1}} ; \quad|g(x)| \leq 2^{n} \alpha, \text { almost everywhere }
$$

and for certain nonoverlapping cubes $Q_{k}$,

$$
b_{k}(x)=0 \quad \text { for } x \notin Q_{k} \text { with } \int_{M} b_{k}(x) d x=0 ; \quad \sum_{k=1}^{\infty} \mu\left|Q_{k}\right| \leq \alpha^{-1}|| f \|_{L^{1}} .
$$

Proof of Proposition 3.4. Since

$$
\{x:|\tilde{m}(P) f(x)|>\alpha\} \subset\{x:|\tilde{m}(P) g(x)|>\alpha / 2\} \cup\{x:|\tilde{m}(P) b(x)|>\alpha / 2\},
$$

notice that $\int_{M}|g|^{2} d x \leq 2^{n} \alpha \int_{M}|g| d x$. Using the $L^{2}$ boundedness of $\tilde{m}(P)$ and Tchebyshev's inequality, we get

$$
\mu\{x:|\tilde{m}(P) g(x)|>\alpha / 2\} \leq C \alpha^{-2}\|g\|_{L^{2}}^{2} \leq C^{\prime} \alpha^{-1}\|f\|_{L^{1}} .
$$


Let $Q_{k}^{*}$ be the cube with the same center as $Q_{k}$ but twice the side-length. After possibly making a translation, we may assume that $Q_{k}=\left\{x: \max \left|x_{j}\right| \leq R\right\}$. Letting $\mathcal{O}^{*}=\bigcup Q_{k}^{*}$, we have $\mu\left|\mathcal{O}^{*}\right| \leq 2^{n} \alpha^{-1}|| f \|_{L^{1}}$ and

$$
\begin{aligned}
\mu\left\{x \notin \mathcal{O}^{*}:|\tilde{m}(P) b(x)|>\alpha / 2\right\} & \leq 2 \alpha^{-1} \int_{x \notin \mathcal{O}^{*}}|\tilde{m}(P) b(x)| d x \\
& \leq 2 \alpha^{-1} \sum_{k=1}^{\infty} \int_{x \notin Q_{k}^{*}}\left|\tilde{m}(P) b_{k}(x)\right| d x .
\end{aligned}
$$

Hence we need only to show that

$$
\int_{x \notin Q_{k}^{*}}\left|\tilde{m}(P) b_{k}(x)\right| d x=\int_{x \notin Q_{k}^{*}}\left|\int_{Q_{k}} K(x, y) b_{k}(y) d y\right| d x \leq C \int_{M}\left|b_{k}\right| d x .
$$

From the double dyadic decomposition (22), we show two estimates of $T_{\lambda, l}(P) b_{k}(x)$ on the set $\left\{x \in M: x \notin \mathcal{O}^{*}\right\}$ :

(I) $\quad\left\|T_{\lambda, l}(P) b_{k}\right\|_{L^{1}\left(x \notin \mathcal{O}^{*}\right)} \leq C\left(2^{l}\right)^{n / 2-s}\left\|b_{k}\right\|_{L^{1}\left(Q_{k}\right)} ;$

(II) $\left\|T_{\lambda, l}(P) b_{k}\right\|_{L^{1}\left(x \notin \mathcal{O}^{*}\right)} \leq C\left(2^{l}\right)^{n / 2-s_{0}}\left[\lambda \max _{y, y_{0} \in Q_{k}} \operatorname{dist}\left(y, y_{0}\right)\right]\left\|b_{k}\right\|_{L^{1}\left(Q_{k}\right)}$.

Given our observation (23), in order to prove $(I),(I I)$, it suffices to show it for all geodesic balls $B_{R_{\lambda, l}}$ of radius $R_{\lambda, l}=2^{l} \lambda^{-1}$. Such bounds are true, as shown in 24] and 25] from Lemma 5.1, Lemma 5.2, and the Hölder inequality. From our observation (23) and $(I)$, for $\operatorname{dist}(x, y) \leq C R$, we have

$$
\begin{aligned}
\sum_{l=-\infty}^{\infty}\left\|T_{\lambda, l}(P) b_{k}\right\|_{L^{1}\left(x \notin \mathcal{O}^{*}\right)} & \leq C \sum_{2^{l} \geq c \lambda \operatorname{dist}(x, y)}\left(2^{l}\right)^{n / 2-s}\left\|b_{k}\right\|_{L^{1}\left(Q_{k}\right)} \\
& \leq C_{s}(\lambda \operatorname{dist}(x, y))^{n / 2-s}\left\|b_{k}\right\|_{L^{1}\left(Q_{k}\right)} \\
& \leq C_{s}(\lambda R)^{n / 2-s}\left\|b_{k}\right\|_{L^{1}\left(Q_{k}\right)},
\end{aligned}
$$

and for $\max _{y, y_{0} \in Q_{k}} \operatorname{dist}\left(y, y_{0}\right) \leq C R$, from $(I I)$ with $n / 2<s_{0}<\min \{s, n / 2+1\}$,

$$
\begin{aligned}
& \sum_{l=-\infty}^{\infty} \quad\left\|T_{\lambda, l}(P) b_{k}\right\|_{L^{1}\left(x \notin \mathcal{O}^{*}\right)} \\
& \quad \leq C \sum_{2^{l} \geq c \lambda \operatorname{dist}(x, y)}\left(2^{l}\right)^{\frac{n}{2}-s_{0}}\left[\lambda \max _{y, y_{0} \in Q_{k}} \operatorname{dist}\left(y, y_{0}\right)\right]\left\|b_{k}\right\|_{L^{1}\left(Q_{k}\right)} \\
& \quad \leq C_{s_{0}}(\lambda \operatorname{dist}(x, y))^{\frac{n}{2}-s_{0}}\left[\lambda \max _{y, y_{0} \in Q_{k}} \operatorname{dist}\left(y, y_{0}\right)\right]\left\|b_{k}\right\|_{L^{1}\left(Q_{k}\right)} \\
& \quad \leq C_{s_{0}}(\lambda R)^{\frac{n}{2}+1-s_{0}}\left\|b_{k}\right\|_{L^{1}\left(Q_{k}\right)} .
\end{aligned}
$$


Therefore, we combine the above two estimates and conclude that

$$
\begin{aligned}
\int_{x \notin Q_{k}^{*}} & \left|\tilde{m}(P) b_{k}(x)\right| d x \\
\leq & \sum_{j=0}^{\infty} \sum_{l=-\infty}^{\infty}\left\|T_{2^{j}, l}(P) b_{k}\right\|_{L^{1}\left(x \notin \mathcal{O}^{*}\right)} \\
\leq & C_{s} \sum_{2^{j} R>1}(\lambda R)^{\frac{n}{2}-s}\left\|b_{k}\right\|_{L^{1}}+C_{s_{0}} \sum_{2^{j} R \leq 1}(\lambda R)^{\frac{n}{2}+1-s_{0}}\left\|b_{k}\right\|_{L^{1}} \\
\leq & C_{s}\left\|b_{k}\right\|_{L^{1}}
\end{aligned}
$$

Hence we have the weak-type $(1,1)$ estimate on the main term

$$
\mu\{x:|\tilde{m}(P) f(x)|>\alpha\} \leq \alpha^{-1}\|f\|_{L^{1}} .
$$

Combining Proposition 3.3 and Proposition 3.4, we prove the sharp Hörmander Multiplier Theorem for the Neumann Laplacian.

\section{ACKNOWLEDGEMENTS}

Partial results of this paper were obtained in the author's Ph.D. thesis [23] at Johns Hopkins University. The author would like to thank his advisor, Professor C. D. Sogge, for bringing the problems to him and for a number of helpful conversations on these problems and his research. The author's research is supported by the National Science Foundation under grants NSF-DMS-0602151 and NSF-DMS0852507.

\section{REFERENCES}

[1] M. Christ and C. D. Sogge, The weak type $L^{1}$ convergence of eigenfunction expansions for pseudo-differential operators. Inv. Math. 94 (1988), 421-453. MR958838 (89j:35096)

[2] X. T. Duong, E. M. Ouhabaz, and A. Sikora, Plancherel-type estimates and sharp spectral multipliers. J. Funct. Anal. 196 (2002), 443-485. MR.1943098 (2003k:43012)

[3] C. Fefferman: Inequalities for strongly singular convolution operators, Acta Math. 124 (1970), 9-36. MR0257819(41:2468)

[4] D. Gilbarg and N. S. Trudinger, Elliptic partial differential equations of second order, Springer-Verlag, 2001 MR 1814364 (2001k:35004)

[5] D. Grieser, $L^{p}$ bounds for eigenfunctions and spectral projections of the Laplacian near concave boundaries, PhD thesis, UCLA, 1992.

[6] D. Grieser, Uniform bounds for eigenfunctions of the Laplacian on manifolds with boundary. Comm. P.D.E. 27 (7-8), 1283-1299 (2002). MR1924468 (2003g:58036)

[7] L. Hörmander, The spectral function of an elliptic operator, Acta Math. 121 (1968), 193-218. MR0609014 (58:29418)

[8] L. Hörmander, The analysis of linear partial differential operators. III, Springer-Verlag, 1985. MR781536 (87d:35002a)

[9] Pham The Lai, Meilleures estimations asymptotiques des restes de la fonction spectrale et des valeurs propres relatifs au laplacien. Math. Scand. 48 (1981), no. 1, 5-38. MR621413 (83b:35127a)

[10] M. H. Protter and H. F. Weinberger, Maximum principles in differential equations, Springer Verlag, 1984. MR762825 (86f:35034)

[11] A. Seeger and C. D. Sogge, On the boundedness of functions of pseudo-differential operators on compact manifolds. Duke Math. J. 59 (1989), 709-736. MR.1046745 (91d:58244)

[12] R. Seeley, An estimate near the boundary for the spectral function of the Laplace operator. Amer. J. Math. 102 (1980), no. 5, 869-902. MR590638 (82k:58097)

[13] H. F. Smith, Spectral cluster estimates for $C^{1,1}$ metrics, Amer. Jour. Math. 128 (2006), 1069-1103. MR:2262171 (2009i:35061) 
[14] H. F. Smith and C. D. Sogge, On the critical semilinear wave equation outside of convex obstacles, J. Amer. Math. Soc. 8 (1995), no. 4, 879-916. MR1308407(95m:35128)

[15] H. F. Smith and C. D. Sogge, On the $L^{p}$ norm of spectral clusters for compact manifolds with boundary. Acta Math. 198 (2007), no. 1, 107-153. MR2316270 (2008d:58026)

[16] C. D. Sogge, On the convergence of Riesz means on compact manifolds. Ann. of Math. (2) 126 (1987), 439-447. MR908154 (89b:35126)

[17] C. D. Sogge, Concerning the $L^{p}$ norm of spectral clusters for second-order elliptic operators on compact manifolds, J. Funct. Anal. 77 (1988), no. 1, 123-138. MR930395 (89d:35131)

[18] C. D. Sogge, Fourier integrals in classical analysis. Cambridge Tracts in Mathematics, 105. Cambridge University Press, Cambridge, 1993. MR.1205579 (94c:35178)

[19] C. D. Sogge, Eigenfunction and Bochner Riesz estimates on manifolds with boundary. Mathematical Research Letter 9, 205-216 (2002). MR1903059 (2003f:58051)

[20] C. D. Sogge and S. Zelditch, Riemannian manifolds with maximal eigenfunction growth. Duke Math. J. 114, no. 3 (2002), 387-437. MR1924569 (2004b:58053)

[21] E. M. Stein and G. Weiss, Introduction to Fourier analysis on Euclidean spaces. Princeton University Press, Princeton, 1971. MR 0304972 (46:4102)

[22] M. Taylor, Pseudodifferential Operators. Princeton Univ. Press, Princeton, NJ, 1981. MR0618463 (82i:35172)

[23] Xiangjin Xu, Eigenfunction Estimates on Compact Manifolds with Boundary and Hörmander Multiplier Theorem. PhD thesis, Johns Hopkins University, May 2004.

[24] Xiangjin Xu, New proof of Hörmander Multiplier Theorem on compact manifolds without boundary. Proc. Amer. Math. Soc. 135 (2007), 1585-1595. MR2276671(2008a:58023)

[25] Xiangjin $\mathrm{Xu}$, Gradient estimate for eigenfunctions on compact manifolds with boundary and Hörmander Multiplier Theorem. Forum Math. 21, no. 3 (2009), 455-476. MR 2526794 (2010d:58026)

Department of Mathematical Sciences, Binghamton University, State University of New York, Binghamton, New York 13902

E-mail address: xxu@math.binghamton.edu 